\title{
"Evaluation of Anti-Inflammatory Effects of Allopurinol in Diabetic Patients with Chronic Kidney Disease (DM-CKD): A Clinical Study"
}

\section{Mahya Daniyali}

Shahid Beheshti University of Medical Sciences

\section{Leila Mahmoudieh}

Shahid Labbafinejad Medical Center, Shahid Beheshti University of Medical Sciences

Golnaz Afzal

Shahid Beheshti University of Medical Sciences

\section{Farzaneh Hematian}

Ahvaz Jondishapur University of Medical Sciences

\section{Omid Moradi}

Shahid Beheshti University of Medical Sciences

Shadi ziaie ( $\nabla$ Shadi_z73@yahoo.com )

Shahid Beheshti University of Medical Sciences

\section{Research Article}

Keywords: Diabetic Nephropathy, Renal Insufficiency Chronic, Allopurinol, Anti-Inflammatory Agents, Uric Acid

Posted Date: June 14th, 2021

DOl: https://doi.org/10.21203/rs.3.rs-585871/v1

License: (c) (i) This work is licensed under a Creative Commons Attribution 4.0 International License. Read Full License 


\section{Abstract}

Background: Diabetic nephropathy is the most prevalent cause of end-stage kidney disease (ESRD). Besides, factors such as; pro-fibrotic, cytokines, vascular endothelial growth factor, inflammatory factor, and uric acid may play a role in creating and progressing diabetic nephropathy. Decreasing the serum level of inflammatory factors can be useful in the treatment of diabetic nephropathy. Therefore, this study aimed to evaluate allopurinol's anti-inflammatory effects in diabetic patients with chronic kidney disease.

Methods: In this clinical trial, 60 diabetic patients with chronic kidney disease and normal uric acid level were enrolled into the study with certain inclusion and exclusion criteria. Patients received allopurinol at a dose of $100 \mathrm{mg}$ daily. Demographic parameters, laboratory results in blood urea nitrogen (BUN), serum creatinine (sCr), glomerular filtration rate (GFR), 24-hour urine protein (PrUrine24h), uric acid, serum albumin (Alb), systolic blood pressure (SBP), diastolic blood pressure (DBP), glycosylated hemoglobin (HbA1C) and high sensitivity $\mathrm{C}$ reactive protein (HSCRP), as well as adverse reactions, were recorded at baseline, ones and three months after.

Results: The results showed that patients were not different in point of demographic parameters at baseline. Laboratory results such as; BUN, sCr, GFR, PrUrine24h, Alb, SBP, DBP, and HbA1C did not change significantly over study duration ( $P>0.05$ ), except uric acid and HSCRP, which were significantly decreased in patients $(P=0.024$ and $P=0.016$, respectively). There was not any notable adverse reaction among patients.

Conclusion: Low dose Allopurinol (100 mg/day) reduced uric acid and inflammatory biomarker (HSCRP) after three administration months. According to the present study results, Allopurinol can be considered an auxiliary, inexpensive, and low side-effect therapy in diabetic patients with chronic kidney disease.

\section{Background}

Diabetic nephropathy is one of the most important causes of chronic kidney disease (CKD) and end-stage kidney disease (ESRD) worldwide in chronic type one and two diabetes mellitus (DM) patients [1, 2]. Approximately 15 years after the beginning of DM, diabetic nephropathy develops in $20 \%$ to $30 \%$ of patients [3, 4]. In 2017, the international diabetes federation estimated that 425 million adults between 20 and 79 have diabetes, corresponding to an $8.8 \%$ prevalence of diabetes globally [5]. CKD is defined as abnormalities of kidney function or structure or an estimated GFR $<60 \mathrm{~mL} / \mathrm{min}$ per $1.73 \mathrm{~m} 2$ present for $>3$ months [6]. The prognosis of CKD patients with lower GFR and higher albuminuria is poor, which indicates a strong relationship with cardiovascular diseases and all-cause mortality [7, 8]. Globally, the incidence of CKD increased by $87 \%$, and the rate of death among CKD patients rose by $98 \%$ from 1990 to 2016 [9].

Researchers show that a chronic state of oxidative stress generally accompanies diabetes. Factors such as pro-fibrotic, cytokines, vascular endothelial growth factor, uric acid, and inflammatory factor are responsible for the progression of complications in macro-and microvascular involvement [10-12]. 
Additionally, various risk factors, including metabolic abnormalities (e.g., hyperglycemia, obesity, hypertension, and dyslipidemia), disease duration, and hemodynamic changes, affect the kidney's structural and functional [13]. Hence, various studies have begun to focus on therapeutic approaches that directly or indirectly target inflammation pathways by suppressing cytokine signaling with specific inhibitors or novel pharmacological [14].

Xanthine oxidase appears to play a key role in vascular dysfunction after intermittent hypoxia exposure. Allopurinol administration, a Xanthine oxidase inhibitor, could reduce the damage of diabetes via its antioxidant effects by decreasing the free radicals and final serum levels of uric acid [15]. However, the impact of allopurinol in modulating the process of diabetic nephropathy has not been well documented.

Concerning the role of uric acid and inflammatory biomarker HSCRP in diabetic patients to cause vascular and glomerular injuries, decreasing GFR, we evaluated the role of allopurinol in diabetic nephropathy. This article aimed to study the anti-inflammatory effects of allopurinol in diabetic patients with chronic kidney disease.

\section{Methods}

Study design

This study is a clinical trial conducted from May 2017 to December 2017 at Shahid Labbafi Nedjad educational Hospital in Iran, Tehran. The Ethics Committee for Research at Shahid Beheshti University of Medical Sciences, Tehran, Iran, approved the study design and procedures with Ethics code: IR.SBMU.PHNM.1395.421

\section{Data collection}

There are, three-stage (baseline, one month, and three months after) in this clinical trial. The study population consisted of adult patients with type 1 or 2 diabetes-induced chronic kidney disease who had an active and fixed medical profile with monthly visits in this hospital. Patients screened for eligibility to participate in the study. The patients on dialysis, known allergy to allopurinol, gout, thrombocytopenia, and who received prednisolone, pioglitazone, vitamin E, and allopurinol used to lower levels of uric acid were excluded. Included subjects had to fulfill the following inclusion criteria: (1) presence of chronic kidney disease, defined as having an estimated GFR lower than $60 \mathrm{ml} / \mathrm{min}$; (2) normal uric acid level; and (3) All of the participants were included after signing informed consent forms. Patients received a dose of $100 \mathrm{mg}$ of allopurinol tablet by mouth once a day for 90 days. The tablet form of allopurinol was obtained from Jalinous Pharmaceutical Company (Iran). During the study, the patients who fulfill the following criteria could not continue to participate in the study: (1) Incidence of allopurinol hypersensitivity skin reaction; (2) Diarrhea; (3) Nausea; (4) Raise liver enzymes; (5) Elevated levels of alkaline phosphatase; (6) Platelet count lower than 100,000 per microliter of blood. Finally, among sixty patients, fifty-six of them satisfied these criteria and were screened; four patients developed skin reactions or nausea during the study, so they were excluded. Patients were followed up monthly until 
three months later. Blood samples and urine from the study participants were collected on days 1, 30, and 90. Portions of the serum and urine were frozen immediately and saved at j70-C. At the end of the study, all frozen samples were mailed to the collaborative laboratory, where biochemical and inflammatory markers were measured.

\section{Definitions of outcomes}

The primary outcome was the evaluation of effect of allopurinol in modulating the process of diabetic nephropathy based on other studies that observed the anti-inflammatory effect of allopurinol in normouremic patients by decreasing oxidative stress [16-18]. The markers tested were as follows: BUN, $\mathrm{sCr}$, uric acid, Alb, HbA1C, HSCRP, and PrUrine24h. The assays carried out in this study are standard ones used in many studies of nephropathy and inflammatory biomarkers. Blood sample and urine were also tested locally (laboratory department in hospital) for kidney function tests and PrUrine24h. Data were also collected from each participant regarding weight, GFR, SBP, DBP, and concomitant medication use.

Statistical analysis

The results were summarized and analyzed using the SPSS v.23.0 software (IBM Corp., Armonk, NY, USA). A P value of $<0.05$ was considered as the significance level. In the analytical statistics section, after determining the pattern of data distribution with the Klomogorov-Smirnov statistical test's help, appropriate statistical tests were selected. Repeat-measure ANOVA was used to examine the differences between measurements. The least significant difference (LSD) test is used in the context of the analysis of variance. For descriptive analysis, means and SDs are reported for continuous variables, and frequencies and percentages are reported for categorical variables.

\section{Results}

In this study, among 60 eligible patients with diabetes-induced nephropathy, 56 were completed the study successfully.

Table 1 presents the descriptive statistical analysis of the collected data from patients. Based on the obtained results, $43(71.7 \%)$ patients were male, and 17 (28.3\%) were female. Participants had a mean age of $60.2 \pm 10.1$ years.

Table 2 demonstrates the mean levels of BUN, Scr, GFR, Proteinuria, uric acid, Alb, SBP, DBP, HgA1c, and HSCRP at baseline, after the one-month and three-months of the study period.

As illustrated in Table 3, the one-sample Kolmogorov-Smirnov test is used to evaluate the data's normality. According to these results $(P<0.05)$, the distribution of data related to these samples in Table 3 is not normal. However, the Kruskal-Wallis statistical test is utilized, a nonparametric (distribution-free) test for quantitative analytical analysis (Table 4). 
Furthermore, to compare these variables at baseline and one and three months after the intervention, the analysis of variance (ANOVA) test is used to compare these variables at the different times two by two LSD post hoc tests used. Table 5 shows no significant difference in the mean scores of all variables except uric acid and HSCRP at baseline and immediately after one and 3-month of intervention $(P>0.05)$.

\section{Discussion}

Diabetic nephropathy (DN) is a chronic kidney disease, which has become one of the leading causes of ESRD, morbidity, and mortality worldwide $[19,20]$.

Allopurinol is frequently used to improve the uric acid profile. It is also reported to reduce diabetic nephropathy, cardiovascular events, and hospitalization via decreasing C-reactive protein and its antioxidant effects [21,22]. However, the efficacy of allopurinol in reducing the level of inflammatory marker HSCRP in individuals with diabetic nephropathy is still controversial.

This study was conducted to answer this question and found that allopurinol could reduce the uric acid and HSCRP during the survey. However, allopurinol has no beneficial effect on kidney function, blood pressure, albumin, and $\mathrm{HbA} 1 \mathrm{C}$ in diabetic nephropathy.

Although the results of this three-month study do not show any effect of allopurinol on kidney function of these patients without hyperuricemia, several studies have described favorable effects of allopurinol to prevent progression CKD in individuals without gout $[23,24]$. So, maybe, the long-term use of allopurinol could prevent more nephropathy development in a patient's diabetic kidney disease.

Our study demonstrates allopurinol does not affect on blood pressure of 56 patients. At the same time, Yuan-Yuei Chen and et al. evaluated the role of uric acid in cardiovascular disorders on 46,561 eligible participants older than 20 years, which showed that a higher uric acid level is significantly associated with an increased risk of incident hypertension [25]. On the other hand, B. H. Santhosh Pai and colleagues demonstrated the benefit of allopurinol on blood pressure control by decreasing blood uric acid level, reducing kidney disease and mortality in patients with CKD. Using data from many participants with diabetic nephropathy in the future study would determine any uric acid effect on blood pressure.

As our results show, Allopurinol effectively reduces uric acid level and marker of inflammation HSCRP in patients with diabetic kidney disease.

In a study done on 6,403 subjects, high serum uric acid level is a risk factor to CKD progress [26]. However, some studies did not show a significant association between uric acid level and decline in kidney function $[27,28]$. This finding could be because these two studies included a majority of patients with later stages of CKD. Therefore, it is most likely that increased serum uric acid level in the CKD's high progressed stage is not associated with the rate of decline in kidney function.

As mention above, a significant association is found between allopurinol use and reduce inflammatory marker, HSCRP. Likewise, several studies regarding the effect of allopurinol on decreasing HSCRP levels 
in CKD [29-31]. It would be related to reducing nitric oxide synthase through allopurinol use, by blocking oxidative stress, preventing the induction of endothelial dysfunction [32]. Due to few studies on assessing the effect of allopurinol on inflammatory markers in diabetic CKD, this study's strength focused on this result, but further investigations are necessary.

As with any study, our study had some limitations. First, only 56 patients with confirmed diabetic nephropathy were studies. Second, it was just a single-center study; it would be interesting that multicenter studies were conducted in other hospitals to evaluate the efficacy of allopurinol in decreasing inflammatory factors leading to improved kidney function in diabetic patients. Finally, more clinical studies with larger sample sizes, higher quality, and strictly randomized control trial studies should be considered in future studies.

\section{Conclusions}

Allopurinol reduces uric acid and HSCRP significantly. However, allopurinol has no beneficial effect on kidney function in type 1 and 2 diabetics patients with nephropathy. Though exciting, our findings still need high-quality and larger studies to confirm allopurinol's benefit in patients with diabetic nephropathy.

\section{Abbreviations}

Alb: Albumin

BUN: Blood urea nitrogen

CKD: chronic kidney disease

DBP: diastolic blood pressure

DM: diabetes mellitus

ESRD: end-stage kidney disease

GFR: glomerular filtration rate

HbA1c: glycosylated hemoglobin

HSCRP: high sensitivity $C$ reactive

PrUrine24h :24-hour urine protein

SBP: systolic blood pressure

$\mathrm{sCr}$ : serum creatinine 


\section{Declarations}

\section{Ethics approval and consent to participate}

The Ethics Committee also approved this study of Shahid Beheshti University of Medical Sciences, Tehran, Iran. Written informed consent was obtained from each patient.

\section{Consent for publication}

Not applicable

\section{Availability of data and materials}

The datasets used and/or analyzed during the current study available from the corresponding author on reasonable request. Questions regarding this process may be directed to Shadi Ziaie as a corresponding author(Shadi_z73@yahoo.com).

\section{Competing interests}

The authors declare that they have no competing interests.

\section{Funding}

This study did not receive any funding.

\section{Author's contributions}

Research idea and study design: ZS, ML. Data acquisition: DM. Data analysis: MO. Statistical analysis: HF. Drafting the article: AG. Supervision of manuscript: ZS, HF, AG. Revision process: ZS, ML, HF, AG, DM. All authors have read and approved the final version of the manuscript.

\section{Acknowledgements}

We thank all patients who participated in our study and those who carried out the extensive clinical and laboratory work in the clinical investigational unit and the renal laboratory at Dept. of Internal Medicine, Shahid Labbafinejad Medical Center, Shahid Beheshti University of Medical Sciences, Tehran, Iran

\section{Author's information}

\section{Affiliations}

Department of clinical pharmacy, school of pharmacy, Shahid Beheshti University of Medical Sciences, Tehran, Iran

Mahya Daniyali, Golnaz Afzal, Omid moradi, and shadi ziaie 
Department of Internal Medicine, Shahid Labbafinejad Medical Center, Shahid Beheshti University of Medical Sciences, Tehran, Iran

Leila Mahmoudieh

Department of clinical pharmacy, school of pharmacy, Ahvaz Jondishapur University of Medical Sciences, Ahvaz, Iran

Farzaneh Hematian

\section{Corresponding author}

Correspondence to Shadi Ziaie

\section{References}

1. Sulaiman, M.K., Diabetic nephropathy: recent advances in pathophysiology and challenges in dietary management. Diabetology \& metabolic syndrome, 2019. 11: p. 7-7.

2. Wang, G., et al., The analysis of risk factors for diabetic nephropathy progression and the construction of a prognostic database for chronic kidney diseases. Journal of Translational Medicine, 2019. 17(1): p. 264.

3. Orchard, T.J., et al., Prevalence of complications in IDDM by sex and duration: Pittsburgh Epidemiology of Diabetes Complications Study II. Diabetes, 1990. 39(9): p. 1116-1124.

4. Otieno, F.C.F., et al., The burden of unrecognised chronic kidney disease in patients with type 2 diabetes at a county hospital clinic in Kenya: implications to care and need for screening. BMC Nephrology, 2020. 21(1): p. 73.

5. Pedersen, M.L., Diabetes care in the dispersed population of Greenland. A new model based on continued monitoring, analysis and adjustment of initiatives taken. International Journal of Circumpolar Health, 2019. 78(sup1): p. 1709257.

6. Inker, L.A., et al., KDOQI US commentary on the $2012 \mathrm{KDIGO}$ clinical practice guideline for the evaluation and management of CKD. Am J Kidney Dis, 2014. 63(5): p. 713-35.

7. Lim, C.C., et al., Chronic kidney disease, cardiovascular disease and mortality: A prospective cohort study in a multi-ethnic Asian population. Eur J Prev Cardiol, 2015. 22(8): p. 1018-26.

8. Nichols, G.A., et al., Kidney disease progression and all-cause mortality across estimated glomerular filtration rate and albuminuria categories among patients with vs. without type 2 diabetes. BMC Nephrology, 2020. 21(1): p. 167. 
9. Xie, Y., et al., Analysis of the Global Burden of Disease study highlights the global, regional, and national trends of chronic kidney disease epidemiology from 1990 to 2016. Kidney Int, 2018. 94(3): p. 567-581.

10. Pérez-Morales, R.E., et al., Inflammation in Diabetic Kidney Disease. Nephron, 2019. 143(1): p. 1216.

11. Donate-Correa, J., et al., Inflammatory cytokines in diabetic nephropathy. Journal of diabetes research, 2015. 2015.

12. Soliman, G.Z., Effect of vitamin $\mathrm{C}$ and/or vitamin E on kidney, liver and brain functions of streptozotocin-induced diabetic rats. The Egyptian Journal of Hospital Medicine, 2013. 53(1): p. 799-808.

13. Tziomalos, K. and V.G. Athyros, Diabetic nephropathy: new risk factors and improvements in diagnosis. The review of diabetic studies: RDS, 2015. 12(1-2): p. 110.

14. Matoba, K., et al., Unraveling the Role of Inflammation in the Pathogenesis of Diabetic Kidney Disease. International journal of molecular sciences, 2019. 20(14): p. 3393.

15. Pasalic, D., N. Marinkovic, and L. Feher-Turkovic, Uric acid as one of the important factors in multifactorial disorders-facts and controversies. Biochemia medica: Biochemia medica, 2012. 22(1): $p$. 63-75.

16. Schlesinger, N. and L. Brunetti. Beyond urate lowering: analgesic and anti-inflammatory properties of allopurinol. in Seminars in arthritis and rheumatism. 2020. Elsevier.

17. Yiginer, O., et al., Allopurinol improves endothelial function and reduces oxidant-inflammatory enzyme of myeloperoxidase in metabolic syndrome. Clin Res Cardiol, 2008. 97(5): p. 334-40.

18. Lai, S.W., C.L. Lin, and K.F. Liao, Case-control study examining the association between allopurinol use and ischemic cerebrovascular disease. J Investig Med, 2019. 67(1): p. 48-51.

19. Elhefnawy, K.A. and A.M. Elsayed, Prevalence of diabetic kidney disease in patients with type 2 diabetes mellitus. The Egyptian Journal of Internal Medicine, 2019. 31(2): p. 149.

20. Fiseha, T. and Z. Tamir, Prevalence and awareness of chronic kidney disease among adult diabetic outpatients in Northeast Ethiopia. BMC Nephrology, 2020. 21(1): p. 129.

21. Kanbay, M., et al., Allopurinol as a Kidney-Protective, Cardioprotective, and Antihypertensive Agent: Hype or Reality? Blood Purification, 2014. 37(3): p. 172-178.

22. George, J., et al., High-Dose Allopurinol Improves Endothelial Function by Profoundly Reducing Vascular Oxidative Stress and Not by Lowering Uric Acid. Circulation, 2007. 114: p. 2508-16. 
23. Paisansinsup, T., M.K. Breitenstein, and J.T. Schousboe, Association between adverse reactions to allopurinol and exposures to high maintenance doses: implications for management of patients using allopurinol. JCR: Journal of Clinical Rheumatology, 2013. 19(4): p. 180-186.

24. Goicoechea, M., et al., Effect of allopurinol in chronic kidney disease progression and cardiovascular risk. Clinical Journal of the American Society of Nephrology, 2010. 5(8): p. 1388-1393.

25. Chen, Y.-Y., et al., The association of uric acid with the risk of metabolic syndrome, arterial hypertension or diabetes in young subjects-An observational study. Clinica Chimica Acta, 2018. 478: p. 68-73.

26. Iseki, K., et al., Significance of hyperuricemia on the early detection of renal failure in a cohort of screened subjects. Hypertens Res, 2001. 24(6): p. 691-7.

27. Nacak, H., et al., Uric acid is not associated with decline in renal function or time to renal replacement therapy initiation in a referred cohort of patients with Stage III, IV and V chronic kidney disease. Nephrol Dial Transplant, 2015. 30(12): p. 2039-45.

28. Nacak, H., et al., Uric acid: association with rate of renal function decline and time until start of dialysis in incident pre-dialysis patients. BMC Nephrol, 2014. 15: p. 91.

29. Roumeliotis, S., et al., Dietary Antioxidant Supplements and Uric Acid in Chronic Kidney Disease: A Review. Nutrients, 2019. 11(8): p. 1911.

30. Goicoechea, M., et al., Effect of Allopurinol in Chronic Kidney Disease Progression and Cardiovascular Risk. Clinical journal of the American Society of Nephrology : CJASN, 2010. 5: p. 1388-93.

31. Sezer, S., et al., Allopurinol reduces cardiovascular risks and improves renal function in pre-dialysis chronic kidney disease patients with hyperuricemia. Saudi Journal of Kidney Diseases and Transplantation, 2014. 25(2): p. 316-320.

32. Gersch, C., et al., Inactivation of nitric oxide by uric acid. Nucleosides, nucleotides \& nucleic acids, 2008. 27(8): p. 967-978.

\section{Tables}

Table 1. Baseline characteristics of patients 
Variable

Age (year)
Mean \pm SD (range), $\mathrm{n} \pm$ $(\%)$

$65.58 \pm 40.47,(44-96)$

Sex

Male $43(71.7 \%)$

Female

Table 2. The mean levels of biochemical indicators and blood pressure at baseline, and within a one and three-month period

\begin{tabular}{|llll|}
\hline Variable & At baseline & After $1^{\text {th }}$ month & After $3^{\text {th }}$ month \\
\hline Blood urea nitrogen $(\mathrm{mg} / \mathrm{dL})$ & $21.63 \pm 7.83$ & $22.37 \pm 6.88$ & $23.26 \pm 7.37$ \\
\hline Serum Creatinine $(\mathrm{mg} / \mathrm{dL})$ & $1.51 \pm 0.30$ & $1.52 \pm 0.33$ & $1.54 \pm 0.37$ \\
\hline Glomerular filtration rate $(\mathrm{ml} / \mathrm{min})$ & $45.81 \pm 7.45$ & $45.55 \pm 8.22$ & $45.21 \pm 8.93$ \\
\hline 24-hour urine protein $(\mathrm{mg} / \mathrm{L} / \mathrm{day})$ & $645.45 \pm 7.45$ & $503.47 \pm 795.55$ & $407.87 \pm 619.61$ \\
\hline Uric acid $(\mathrm{mg} / \mathrm{dl})$ & $5.19 \pm 0.89$ & $5.02 \pm 0.84$ & $4.83 \pm 0.86$ \\
\hline Albumin $(\mathrm{g} / \mathrm{dl})$ & $4.31 \pm 0.30$ & $4.25 \pm 0.36$ & $4.24 \pm 0.36$ \\
\hline Systolic blood pressure $(\mathrm{mmHg})$ & $132.85 \pm 21.86$ & $131.45 \pm 16.95$ & $130.93 \pm 19.18$ \\
\hline Diastolic blood pressure $(\mathrm{mmHg})$ & $73.10 \pm 9.02$ & $74.88 \pm 8.56$ & $74.50 \pm 10.31$ \\
\hline Glycated hemoglobin $(\%)$ & $7.60 \pm 1.70$ & $7.46 \pm 1.71$ & $7.32 \pm 1.62$ \\
\hline High-sensitivity C-reactive protein $(\mathrm{mg} / \mathrm{dL})$ & $3.63 \pm 1.09$ & $3.27 \pm 0.89$ & $3.22 \pm 0.78$ \\
\hline
\end{tabular}

Table 3. One sample Kolmogorov-Smirnov test for testing distribution 


\begin{tabular}{|lc|}
\hline Variable & P-value \\
\hline Blood urea nitrogen & $<0.001$ \\
\hline Serum Creatinine & $<0.001$ \\
\hline Glomerular filtration rate & 0.034 \\
\hline 24-hour urine protein & $<0.001$ \\
\hline Uric acid & $<0.001$ \\
\hline Albumin & $<0.001$ \\
\hline Systolic blood pressure & $<0.001$ \\
\hline Diastolic blood pressure & $<0.001$ \\
\hline Glycated hemoglobin & $<0.001$ \\
\hline High-sensitivity C-reactive protein & $<0.001$ \\
\hline
\end{tabular}

Table 4. Assessment of the distribution of biochemical indicators and blood pressure during the whole treatment period using the Kruskal-Wallis test 


\begin{tabular}{|c|c|c|c|}
\hline Null Hypothesis & Test & Sig. & Decision \\
\hline $\begin{array}{l}\text { The distribution of Blood urea nitrogen is the } \\
\text { same across of study }\end{array}$ & $\begin{array}{l}\text { one-sample } \\
\text { Kolmogorov-Smirnov } \\
\text { test }\end{array}$ & 0.329 & $\begin{array}{l}\text { Retain the } \\
\text { null } \\
\text { hypothesis }\end{array}$ \\
\hline $\begin{array}{l}\text { The distribution of serum creatinine is the same } \\
\text { across of study }\end{array}$ & $\begin{array}{l}\text { one-sample } \\
\text { Kolmogorov-Smirnov } \\
\text { test }\end{array}$ & 0.997 & $\begin{array}{l}\text { Retain the } \\
\text { null } \\
\text { hypothesis }\end{array}$ \\
\hline $\begin{array}{l}\text { The distribution of glomerular filtration rate is the } \\
\text { same across of study }\end{array}$ & $\begin{array}{l}\text { one-sample } \\
\text { Kolmogorov-Smirnov } \\
\text { test }\end{array}$ & 0.999 & $\begin{array}{l}\text { Retain the } \\
\text { null } \\
\text { hypothesis }\end{array}$ \\
\hline $\begin{array}{l}\text { The distribution of } 24 \text {-hour urine protein is the } \\
\text { same across of study }\end{array}$ & $\begin{array}{l}\text { one-sample } \\
\text { Kolmogorov-Smirnov } \\
\text { test }\end{array}$ & 0.310 & $\begin{array}{l}\text { Retain the } \\
\text { null } \\
\text { hypothesis }\end{array}$ \\
\hline $\begin{array}{l}\text { The distribution of uric acid is the same across of } \\
\text { study }\end{array}$ & $\begin{array}{l}\text { one-sample } \\
\text { Kolmogorov-Smirnov } \\
\text { test }\end{array}$ & 0.019 & $\begin{array}{l}\text { Reject the } \\
\text { null } \\
\text { hypothesis }\end{array}$ \\
\hline $\begin{array}{l}\text { The distribution of albumin is the same across of } \\
\text { study }\end{array}$ & $\begin{array}{l}\text { one-sample } \\
\text { Kolmogorov-Smirnov } \\
\text { test }\end{array}$ & 0.657 & $\begin{array}{l}\text { Retain the } \\
\text { null } \\
\text { hypothesis }\end{array}$ \\
\hline $\begin{array}{l}\text { The distribution of systolic blood pressure is the } \\
\text { same across of study }\end{array}$ & $\begin{array}{l}\text { one-sample } \\
\text { Kolmogorov-Smirnov } \\
\text { test }\end{array}$ & 0.925 & $\begin{array}{l}\text { Retain the } \\
\text { null } \\
\text { hypothesis }\end{array}$ \\
\hline $\begin{array}{l}\text { The distribution of diastolic blood pressure is the } \\
\text { same across of study }\end{array}$ & $\begin{array}{l}\text { one-sample } \\
\text { Kolmogorov-Smirnov } \\
\text { test }\end{array}$ & 0.522 & $\begin{array}{l}\text { Retain the } \\
\text { null } \\
\text { hypothesis }\end{array}$ \\
\hline $\begin{array}{l}\text { The distribution of Glycated hemoglobin is the } \\
\text { same across of study }\end{array}$ & $\begin{array}{l}\text { one-sample } \\
\text { Kolmogorov-Smirnov } \\
\text { test }\end{array}$ & 0.617 & $\begin{array}{l}\text { Retain the } \\
\text { null } \\
\text { hypothesis }\end{array}$ \\
\hline $\begin{array}{l}\text { The distribution of High-sensitivity C-reactive } \\
\text { protein is the same across of study }\end{array}$ & $\begin{array}{l}\text { one-sample } \\
\text { Kolmogorov-Smirnov } \\
\text { test }\end{array}$ & 0.114 & $\begin{array}{l}\text { Retain the } \\
\text { null } \\
\text { hypothesis }\end{array}$ \\
\hline
\end{tabular}

Asymptotic significances are displayed. The significance level is 0.05

Table 5. Paired comparison of the mean levels of biochemical indicators and blood pressure at different times 


\begin{tabular}{|c|c|c|}
\hline Variable & Time & $P$ value \\
\hline \multirow[b]{3}{*}{ Blood urea nitrogen (mg/dL) } & Baseline and immediately after one month & 0.585 \\
\hline & Baseline and immediately after three months & 0.227 \\
\hline & after one month and after three months & 0.507 \\
\hline \multirow[t]{3}{*}{ Serum Creatinine (mg/dL) } & Baseline and immediately after one month & 0.853 \\
\hline & Baseline and immediately after three months & 0.689 \\
\hline & after one month and after three months & 0.829 \\
\hline \multirow[t]{3}{*}{ Glomerular filtration rate (ml/min) } & Baseline and immediately after one month & 0.864 \\
\hline & Baseline and immediately after three months & 0.694 \\
\hline & after one month and after three months & 0.824 \\
\hline \multirow[t]{3}{*}{ 24-hour urine protein (mg/L/day) } & Baseline and immediately after one month & 0.406 \\
\hline & Baseline and immediately after three months & 0.165 \\
\hline & after one month and after three months & 0.576 \\
\hline \multirow[t]{3}{*}{ Uric acid (mg/dl) } & Baseline and immediately after one month & 0.287 \\
\hline & Baseline and immediately after three months & 0.024 \\
\hline & after one month and after three months & 0.225 \\
\hline \multirow[t]{3}{*}{ Albumin (g/dl) } & Baseline and immediately after one month & 0.289 \\
\hline & Baseline and immediately after three months & 0.259 \\
\hline & after one month and after three months & 0.945 \\
\hline \multirow[t]{3}{*}{ Systolic blood pressure (mmHg) } & Baseline and immediately after one month & 0.694 \\
\hline & Baseline and immediately after three months & 0.884 \\
\hline & after one month and after three months & 0.590 \\
\hline \multirow[t]{3}{*}{ Diastolic blood pressure (mmHg) } & Baseline and immediately after one month & 0.296 \\
\hline & Baseline and immediately after three months & 0.412 \\
\hline & after one month and after three months & 0.822 \\
\hline \multirow[t]{3}{*}{ Glycated hemoglobin (\%) } & Baseline and immediately after one month & 0.637 \\
\hline & Baseline and immediately after three months & 0.359 \\
\hline & after one month and after three months & 0.656 \\
\hline High-sensitivity C-reactive protein $(\mathrm{mg} / \mathrm{dL})$ & Baseline and immediately after one month & 0.037 \\
\hline
\end{tabular}

Page 14/15 
Every entry written in boldface in the aforementioned table is of statistical significance $(p<0.05)$ 